\title{
Correlation between MTA2 overexpression and tumour progression in esophageal squamous cell carcinoma
}

\author{
Y.P. LIU ${ }^{1}$, B.E. SHAN ${ }^{2}$, X.L. WANG ${ }^{1}$ and L. MA ${ }^{3}$ \\ ${ }^{1}$ Department of Pathology; ${ }^{2}$ Scientific Research Center; ${ }^{3}$ Department of Surgery, Institute of the Fourth Hospital \\ of Hebei Medical University, Shijiazhuang, Hebei 050011, P.R. China
}

Received December 2, 2011; Accepted January 13, 2012

DOI: $10.3892 /$ etm.2012.475

\begin{abstract}
The aim of this study was to clarify the clinicopathological outcome and prognostic significance of metastasis-associated gene 2 (MTA2) in esophageal squamous cell carcinoma (ESCC). Immunohistochemical staining for MTA2 was performed on surgical specimens obtained from 162 patients with ESCC. Relationships between MTA2 expression and clinicopathological factors, including prognosis, were analyzed. Significant correlations were noted among MTA2 overexpression and TNM clinical classification staging, depth of invasion, presence of regional lymph node metastasis, presence of distant metastasis, lymphatic invasion, bloodvessel invasion and the 5-year survival rates. The expression of MTA2 protein was correlated with tumour progression. Patients with MTA2 overexpression tended to have poor prognosis compared to patients with MTA2 underexpression.
\end{abstract}

\section{Introduction}

Although effective surgical and radiation treatment exist for clinically localized esophageal cancer, refractory metastatic esophageal cancer remains incurable. Distinct sets of genes and proteins dictate the progression from precursor lesions to localized disease and finally to metastatic disease (1). Identifying and characterizing key genes that regulate the metastatic ability of esophageal cancer may help identify which tumours are on an aggressive path from the outset and may aid in treating them adequately before the development of metastases. Through gene expression profiling studies, metastasis-associated gene 2 (MTA2), a gene involved in the transcriptional silencing machinery of mammalian cells, was recently identified to be significantly overexpressed in metastatic prostate cancer when compared to clinically localized disease (2). MTA2 was identified by differential cDNA

Correspondence to: Dr Li Ma, Department of Surgery, Institute of the Fourth Hospital of Hebei Medical University, No. 12 Jiankangt Road, Shijiazhuang, Hebei 050011, P.R. China

E-mail: mali1021@126.com

Key words: metastasis-associated gene 2, esophageal cancer, prognosis, immunohistochemistry library screening of metastatic breast cancer cell lines and confirmed in breast cancer tissue (3). MTA2 overexpression at the transcription level was observed in other cancers, such as gastrointestinal cancers, and was associated with tumour invasiveness and metastasis (4-6). In addition, inhibition of MTA2 protein expression resulted in growth inhibition of cancer cell lines (7). However, the expression of MTA2 in the development and progression of esophageal carcinoma are not clear. In the present study, we characterized for the first time the expression of MTA2 in esophageal cancer progression. All observations suggest that MTA2 is associated with carcinogenesis, progression and metastasis in human tumours.

Based on the above considerations, we performed an immunohistochemical study on MTA2 expression in esophageal squamous cell carcinoma (ESCC) patients, and examined whether any relationships exist among the expression of MTA2, pathological tumour variables and prognosis in patients with ESCC.

\section{Patients and methods}

Patient selection. Surgical specimens were obtained from 162 patients (112 males; 50 females) who had ESCC and underwent potentially curative surgery at the Fourth Hospital of Hebei Medical University (Shijiazhuang, China) between 1996 and 2002. The age of the patients ranged from 30 to 89 years, with a mean of 61.9 years. The tumour stage and disease grade were classified according to the 5 th edition of the TNM Classification of the International Union Against Cancer (8). The evaluation of tumour differentiation was based on histological criteria by the Japanese Society for Esophageal Diseases (9). None of the patients had received irradiation or chemotherapy prior to surgery. Patients who underwent noncurative surgery and/or who had received inadequate follow-up were excluded from this study (10). The information was entered into a database following the approval of the Fourth Hospital of Hebei Medical Univercity Institutional Review Board. M1 tumours were attributable to distant lymph node metastases. Post-operative chemotherapy and/or radiation therapy were not performed until recurrence of the tumour was confirmed by a radiologic or endoscopic examination. Specimens were fixed in $10 \%$ formaldehyde solution and embedded in paraffin. We examined sections that contained both a tumour portion and non-cancerous esophageal epithelium. 
Immunohistochemical staining and evaluation. Immunohistochemical staining of the section for MTA2 was performed by streptoavidin-biotin methods. Antigen retrieval was achieved by boiling the specimen in a citrate buffer $0.01 \mathrm{M}(\mathrm{pH} 7.0)$ at $120^{\circ} \mathrm{C}$ for $3 \mathrm{~min}$, continued with a blocking treatment by normal rabbit serum for $30 \mathrm{~min}$. For MTA2, the specimens were incubated with mouse anti-human monoclonal antibody (sc-55566; Santa Cruz, CA, USA) at a dilution of 1:100 in phosphate-buffered saline (PBS) at $4^{\circ} \mathrm{C}$ overnight. After washing with PBS, specimens were incubated with a secondary antibody for $30 \mathrm{~min}$. Immunohistochemistry was performed using a Histofine SAB-PO (M) kit (Zhongshan, Beijing, China). Human breast tumour expressing MTA2 was used as a positive control. Negative controls were prepared by substituting normal mouse serum for primary antibody; no detectable staining was evident.

The expression of MTA2 in tumour cells was compared to MTA2 expression in non-cancerous epithelium. When the staining in tumour cells (in the cancer cell nests) was stronger than the staining noted in the non-cancerous epithelium, the sample was classified as having MTA2 overexpression; when staining in the tumour cell was as strong as the staining noted in the non-cancerous epithelium it was considered to have weak expression, or when there was no staining at all, the sample was classified as exhibiting MTA2 underexpression. The sections were evaluated independently by three investigators (Y.P. L., G.X. W. and X.L. W.) without knowledge of the clinical and pathological background of the patients. When the interpretation differed among observers, re-evaluation was carried out for a final decision on a conference microscope.

Statistical analysis. Statistical analysis was performed using the unpaired two-group t-test for age. A Chi-square test was used for gender, differentiation, TNM clinical classification, stage and location. In this study, the correlation among MTA2, lymphatic invasion, infiltrative growth pattern and blood-vessel invasion to the tumour tissues was also assessed. The survival curves of the patients were calculated using the Kaplan-Meier method, and analysis was performed using the $\log$-rank test. Differences were considered statistically significant at $\mathrm{P}<0.05$.

\section{Results}

Immunohistochemistry of MTA2 expression. Immunostaining of MTA2 in non-cancerous tissue was detected in the nucleus of the parabasal layer (Fig. 1A). In tumour tissue, MTA2 expression was observed in the nucleus, particularly in cells located in the central layer of the cancer cell nests (Fig. 1B). MTA2 overexpression was detected in 65 of the 162 patients (40.1\%). Ninety-seven cases (59.9\%) were classified as having MTA2 underexpression (Fig. 1C).

Relationship between MTA2 expression and clinicopathological outcome. The relationship between MTA2 and clinicopathological outcome in ESCC is summarized in Table I. There were significant correlations between the overexpression of MTA2 and the TNM clinical classification (depth of invasion, $\mathrm{P}=0.018$; presence of regional lymph node
$\mathbf{A}$

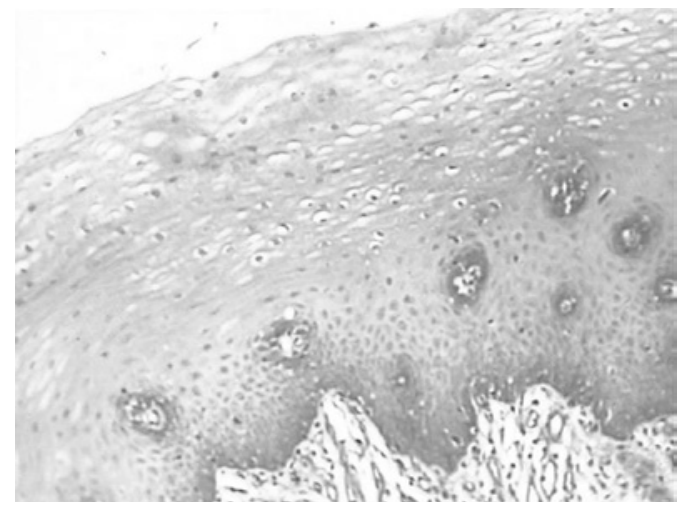

B

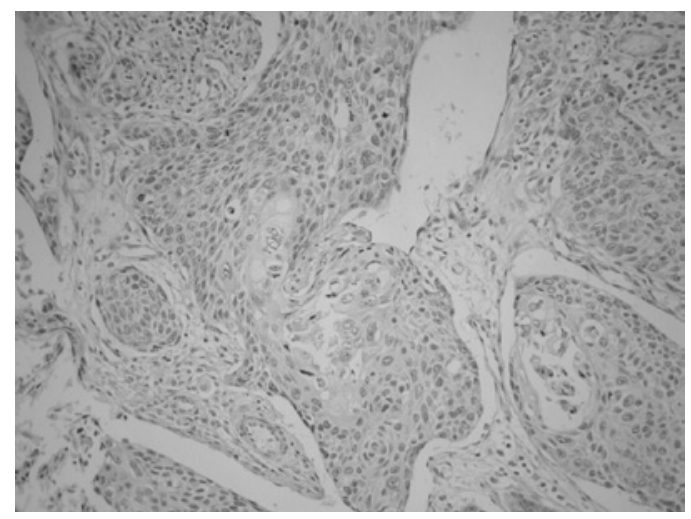

C

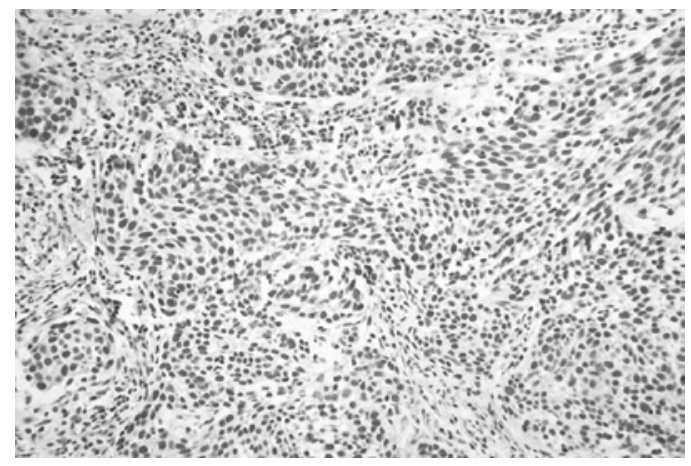

Figure 1. Representative photomicrographs of tissue sections immunostained for MTA2. (A) MTA2 protein was detected in the nucleus in normal esophageal squamous epithelium in the basal layer (magnification, x200). (B) Primary esophageal cancer with MTA2 underexpression in a cancer cell nest (magnification, x400). (C) Primary esophageal cancer with MTA2 overexpression in a cancer cell nest (magnification, $\mathrm{x} 400$ ).

metastasis, $\mathrm{P}=0.009$; presence of distant metastasis, $\mathrm{P}=0.003$; staging, $\mathrm{P}=0.006)$, differentiation $(\mathrm{P}=0.05)$, lymphatic invasion $(\mathrm{P}=0.002)$ and blood-vessel invasion $(\mathrm{P}=0.004)$. However, there was no correlation with patient age, gender and location of the tumours.

Prognostic significance of MTA2 expression. The 5-year survival rates of the ESCC patients with MTA2 overexpression were significantly lower than those with MTA2 underexpression ( $\mathrm{P}=0.002$; Fig. 2). The 5-year survival rates of the patients with overexpression and underexpression of MTA2 were 18.4 and $46.0 \%$, respectively. To further investigate the influence of MTA2 expression within homogeneous stage groups, adding more prognostic sense, we analysed the seperate Kaplan-Meier diagrams for T1, T2, T3, T4 and N0/ 
Table I. Clinicopathological findings and MTA2 expression.

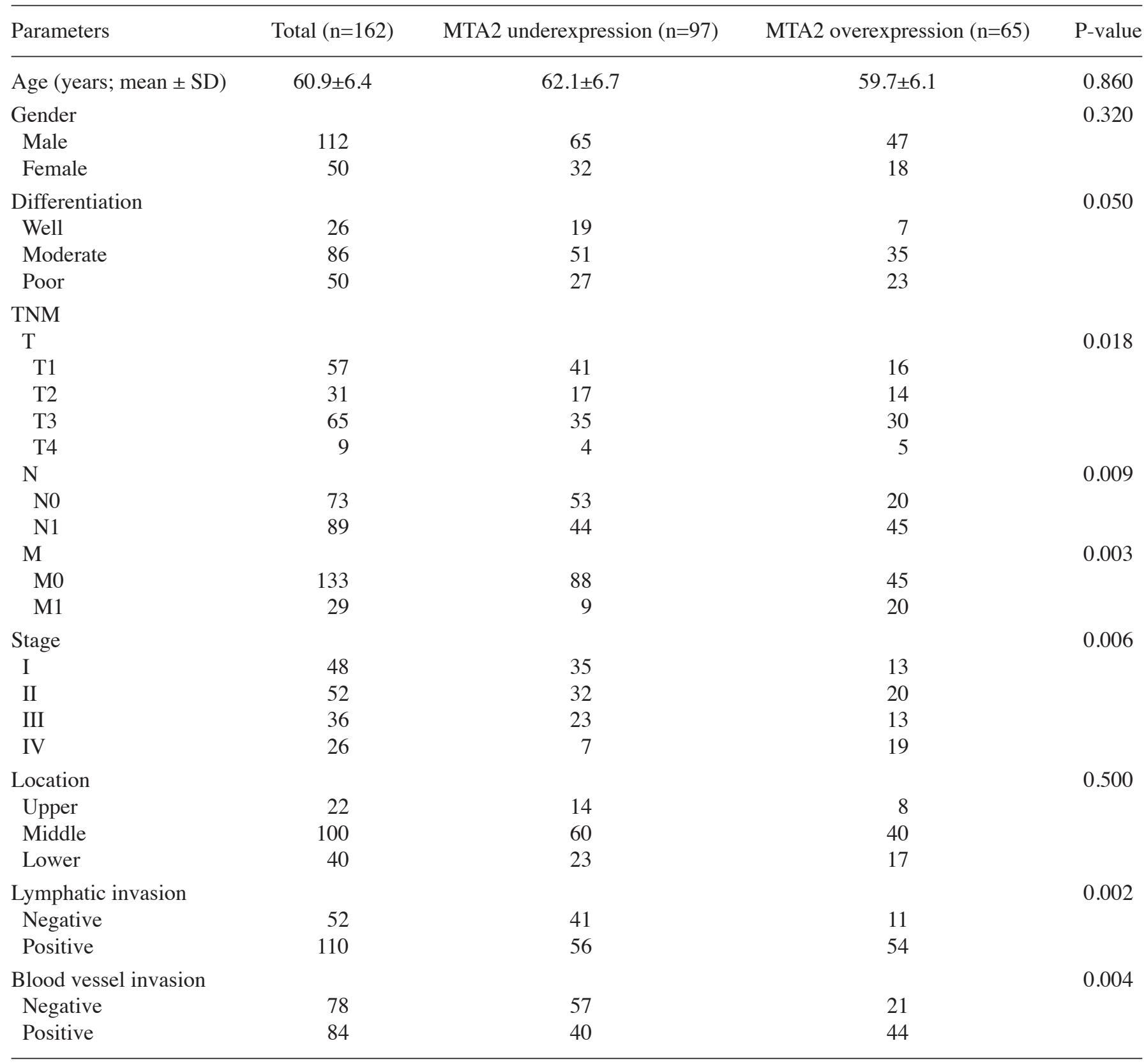

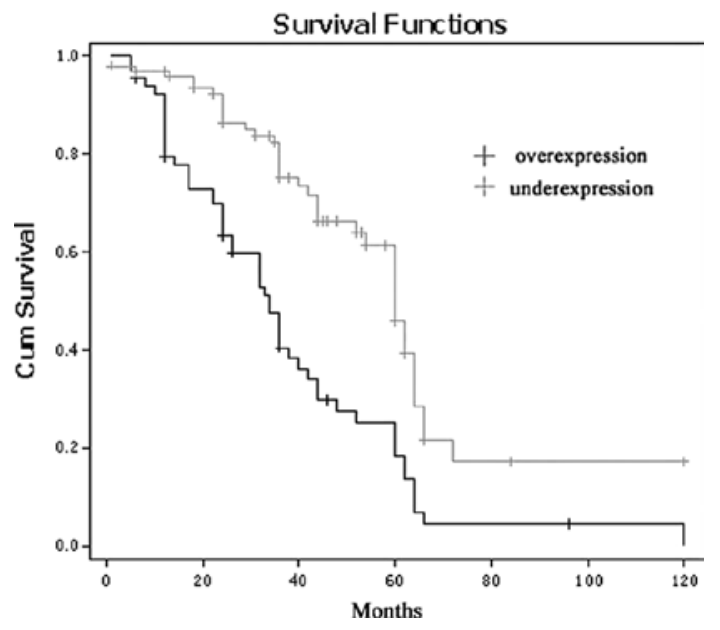

Figure 2. Kaplan-Meier curve demonstrating overall survival for patients according to MTA2 expression.
N1 tumours with MTA2 expression; it was also shown that the 5-year survival rates of ESCC patients with MTA2 overexpression were poorer in every group $(\mathrm{P}=0.036,0.012,0.014$, 0.038 and $0.000,0.002$, respectively; Figs. 3 and 4 ).

\section{Discussion}

MTA2 expression and prognosis in cancer in comparison with previous studies. In this study, we showed that MTA2 overexpression frequently occurred in ESCC and was correlated with several aspects of tumour progression summarized in the TNM classification. Recently, the expression of MTA2 in various types of malignant tumours has been reported. However, only a few reports, including our previous article, showed the prognostic relevance of MTA2 expression in ESCC. Zhang et al (11) reported that MTA2 was expressed in $10 \%$ of epithelial cells with a weak immunoreactivity in the 
A

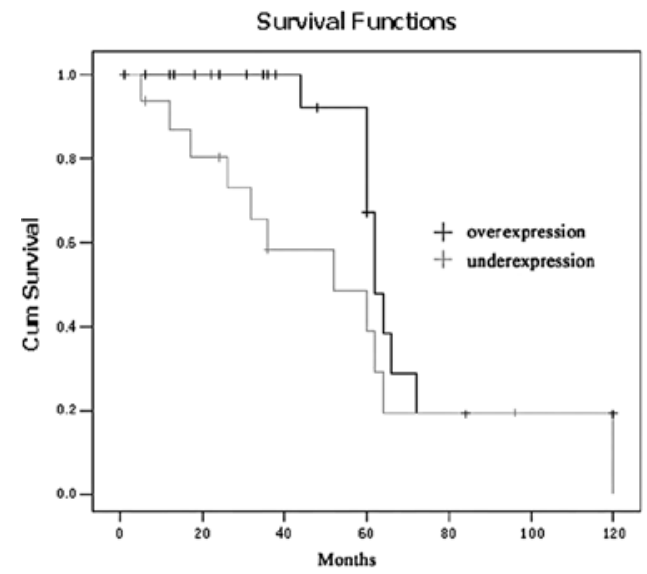

C

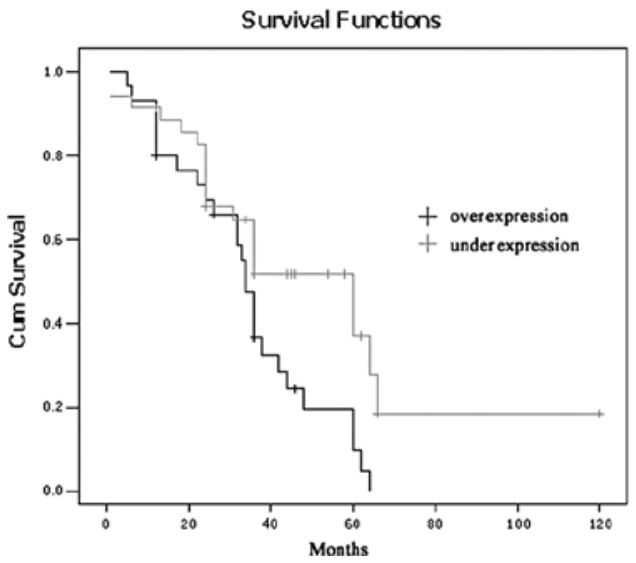

B

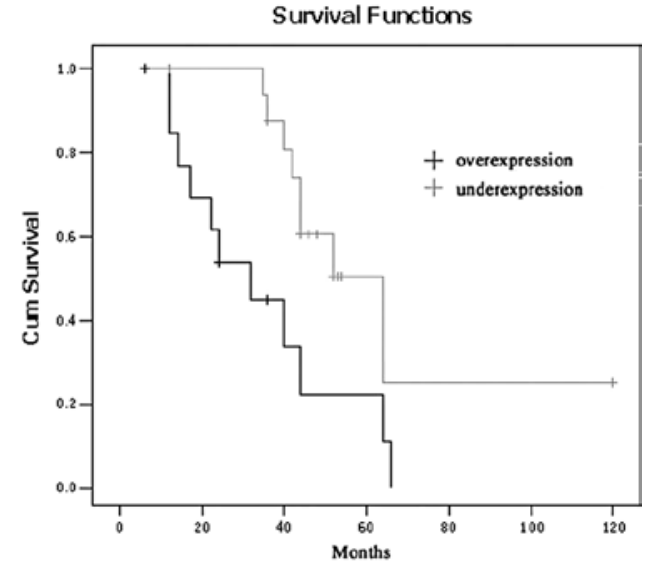

D

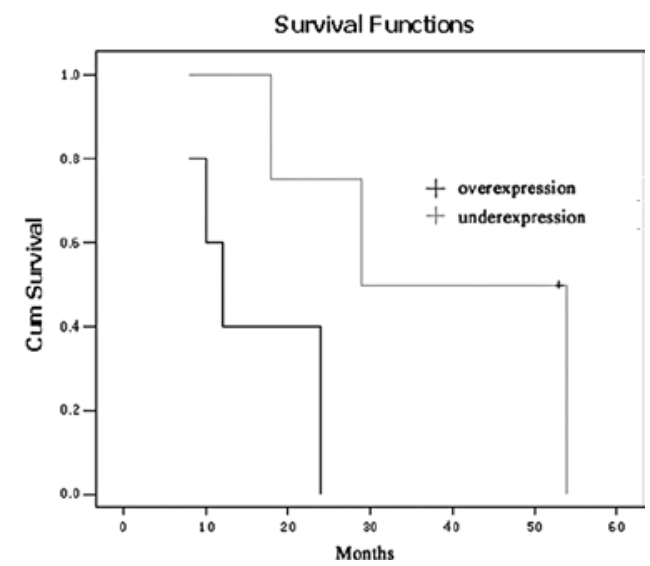

Figure 3. Kaplan-Meier curve demonstrating overall survival of patients by MTA2 expression according to stage. (A) T1, (B) T2, (C) T3 and (D) T4.

A

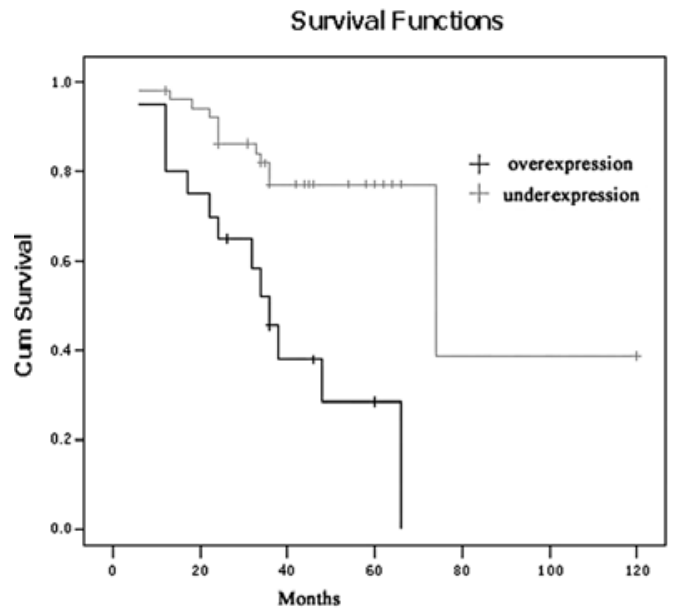

B

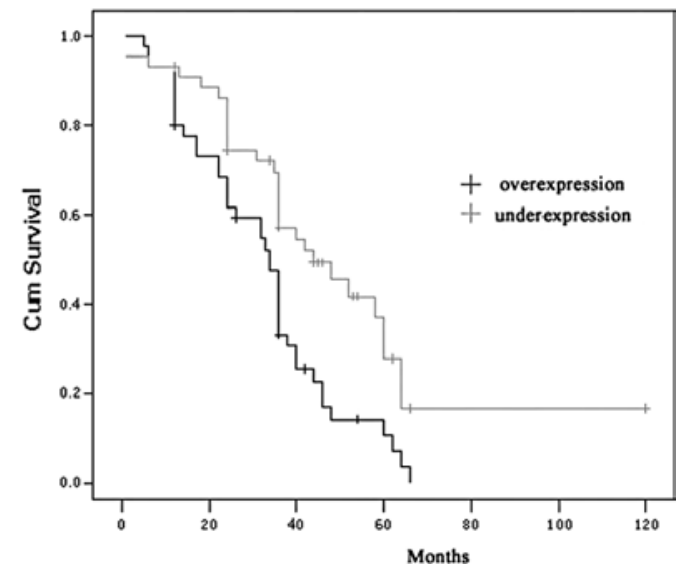

Figure 4. Kaplan-Meier curve demonstrating overall survival of patients by MTA2 expression according to N group. (A) N0 and (B) N1.

nuclear compartment. A marked increase in the nuclear MTA signal was observed in late stage carcinoma. In their study, MTA immunoreactivity was associated with high tumour grade. We previously showed the prognostic relevance of MTA2 expression in a large series of gastric carcinoma and its significant relation to clinicopathological findings. These results indicate that MTA2 overexpression may be an outcome of some important genetic and/or epigenetic change in cancer progression; however, it remains unknown whether in these carcinomas MTA2 expression merely represents a surrogate marker for prognosis or whether it plays a pathogenic role in carcinogenesis and tumour progression.

In non-cancerous epithelial esophageal squamous cells, only slight MTA2 expression was observed in the parabasal layers. MTA2 overexpression was detected in the nucelus of cancer cells, especially in the central layers of the cancer cell nests. These immunohistochemical results suggest that the overexpression of MTA2 protein is correlated with TNM 
clinical classifications (depth of invasion, presence of regional lymph node metastasis, presence of distant metastasis and staging), lymphatic invasion and blood-vessel invasion. There was a statistically significant correlation between MTA2 overexpression and patient survival.

The prognosis of patients with overexpression of MTA2 was poorer than that of patients with MTA2 underexpression. Cancer invasion and metastasis are complex processes that include alterations in cell adhesion, allowing transformed cells to invade and migrate (12-14). MTA proteins are physiologically expressed at only low levels in human tissue, except the testis (10). Its expression has been found to be associated with progression in solid cancers of various organs and cancer cell lines with high invasive potential (15) and thus overexpression of MTA protein has been reported to be correlated with tumour invasiveness and lymph node metastasis in ESCC. Further study is required to elucidate the relationship between MTA2 and prognosis in ESCC.

In this study, patients with MTA2 overexpression were found to have a poorer prognosis for a 5-year survival. However, by multivariate analysis, MTA2 overexpression did not appear to be an independent prognostic factor. It may be influenced by the depth of tumour invasion and distant metastasis. In esophageal carcinoma, the presence of lymph node metastasis and the number of lymph node metastases were associated with the worst prognosis. As MTA2 overexpression was related to lymph node metastases, patients with MTA2 overexpression had a poor prognosis.

In conclusion, according to our data, MTA2 is usually overexpressed in ESCC cells compared to non-cancerous esophageal epithelia, and increased expression of MTA2 was associated with a poor prognosis. Thus, our findings suggest that MTA2 overexpression may play an important role in the progression of ESCC. Tumour-specific MTA2 down-regulation may become a novel therapeutic strategy for ESCC patients.

\section{Acknowledgements}

This study was supported by a grant from the Health Department of Hebei Province (No. 07120), and a grant from the Hebei Province Natural Science Foundation of P.R. China (No. C2009001209).

\section{References}

1. Shappell SB, Olson SJ, Hannah SE, et al: Elevated expression of 12/15-lipoxygenase and cyclooxygenase- 2 in a transgenic mouse model of prostate carcinoma. Cancer Res 63: 2256-2267, 2003.

2. Toh Y, Kuninaka S, Endo K, et al: Molecular analysis of a candidate metastasis-associated gene, MTA1: possible interaction with histone deacetylase 1. J Exp Clin Cancer Res 19: 105-111, 2000.

3. Cui Y, Niu A, Pestell R, et al: Metastasis-associated protein 2 is a repressor of estrogen receptor alpha whose overexpression leads to estrogen-independent growth of human breast cancer cells. Mol Endocrinol 20: 2020-2035, 2006.

4. Nicolson GL, Nawa A, Toh Y, et al: Tumor metastasis-associated human MTA1 gene and its MTA1 protein product: role in epithelial cancer cell invasion, proliferation and nuclear regulation. Clin Exp Metastasis 20: 19-24, 2003.

5. Saito $\mathrm{M}$ and Ishikawa F: The mCpG-binding domain of human MBD3 does not bind to $\mathrm{mCpG}$ but interacts with $\mathrm{NuRD} / \mathrm{Mi} 2$ components HDAC1 and MTA2. J Biol Chem 277: 35434-35439, 2002.

6. Wong JJ, Hawkins NJ and Ward RL: Colorectal cancer: a model for epigenetic tumorigenesis. Gut 56: 140-148, 2007.

7. Yao YL and Yang WM: The metastasis-associated proteins 1 and 2 form distinct protein complexes with histone deacetylase activity. J Biol Chem 278: 42560-42568, 2003.

8. Kumar R, Wang RA and Bagheri-Yarmand R: Emerging roles of MTA family members in human cancers. Semin Oncol 30: 30-37, 2003.

9. Liu YP, Ma L, Wang SJ, et al: Prognostic value of lymph node metastases and lymph node ratio in esophageal squamous cell carcinoma. EJSO 36: 155-159, 2010.

10. Zhang H, Stephens C and Kumar R: Metastasis tumor antigen family proteins during breast cancer progression and metastasis in a reliable mouse model for human breast cancer. Clin Cancer Res 12: 1479-1486, 2006.

11. Fujita N, Kajita M, Taysavang P, et al: Hormonal regulation of metastasis-associated protein 3 transcription in breast cancer cells. Mol Endocrinol 18: 2937-2949, 2004.

12. Park SI, Zhang J, Phillips KA, et al: Targeting SRC family kinases inhibits growth and lymph node metastases of prostate cancer in an orthotopic nude mouse model. Cancer Res 68: 3323-3333, 2008.

13. Manavathi B, Singh K and Kumar R: MTA family of coregulators in nuclear receptor biology and pathology. Nucl Recept Signal 5: e010, 2007.

14. Li W, Zhang J, Liu X, Xu R and Zhang Y: Correlation of appearance of metastasis-associated protein1 (Mta1) with spermatogenesis in developing mouse testis. Cell Tissue Res 329: 351-362, 2007.

15. Adjei AA: Pemetrexed in the treatment of selected solid tumors. Semin Oncol 29: 50-53, 2002. 